\title{
Establishment of an experimental glaucoma animal model: A comparison of microbead injection with or without hydroxypropyl methylcellulose
}

\author{
HANHAN LIU and CHUN DING \\ Department of Ophthalmology, The Second Xiangya Hospital of Central South University, \\ Changsha, Hunan 410011, P.R. China
}

Received April 18, 2016; Accepted March 31, 2017

DOI: $10.3892 /$ etm.2017.4728

\begin{abstract}
The present study aimed to compare microbead injection with and without hydroxypropyl methylcellulose (HPM) in order to establish an experimental animal model of glaucoma. This model was established in C57BL/6 mice and transgenic mice expressing cyan fluorescent protein (CFP) under the control of the Thyl promoter in retinal ganglion cells (RGCs). C57BL/6 mice aged between 12 and 20 weeks old were randomly separated into three groups, which received different injections into the anterior chamber of the eye. Group A (microbead) received $2 \mu 1$ microbeads $\left(10 \times 10^{6}\right.$ beads $\left./ \mathrm{ml}\right)$ and $1 \mu \mathrm{l}$ air. Group B (microbeads + HPM) received $2 \mu \mathrm{l}$ microbeads and $1 \mu \mathrm{l}$ HPM. Group C (control group) received $2 \mu 1$ PBS and $1 \mu 1$ air. The intraocular pressure (IOP) was measured with a tonometer under topical anesthesia daily for 1 month. A single injection of microbeads, with or without HPM, induced consistent IOP elevation when compared with the control group. Thyl-CFP mice received an injection of $2 \mu \mathrm{l}$ microbeads and $1 \mu \mathrm{l}$ HPM into the anterior chamber of the eyes, and the number of $\mathrm{CFP}^{+} \mathrm{RGCs}$ was subsequently assessed in vivo by confocal scanning laser microscopy in the same area of the retina weekly for 6 weeks. The results from in vivo imaging of Thyl-CFP mice were comparable with the immunohistochemical staining results from the C57BL/6 mice. The combined injection of microbeads and HPM induced longer and higher peaks of IOP elevation when compared with the microbeads alone. The rate of RGC loss following the administration of microbeads alone was $25.0 \pm 1.3 \% 6$ weeks after the initial IOP elevation, while it was $33.2 \pm 1.9 \%$ following the administration of microbeads + HPM. These results indicate that the injection of microbeads + HPM is a more effective
\end{abstract}

Correspondence to: Dr Chun Ding, Department of Ophthalmology, The Second Xiangya Hospital of Central South University, 139 Middle Renmin Road, Changsha, Hunan 410011, P.R. China E-mail: 2107407161@qq.com

Key words: glaucoma, retinal ganglion cell, microbead, hydroxypropyl methylcellulose, intraocular pressure, animal model method of establishing a mouse model with chronic elevation of IOP. In addition, the in vivo imaging that can be used with this technique provides an effective and noninvasive approach for monitoring the progress of RGC loss.

\section{Introduction}

Glaucoma, a leading cause of blindness worldwide, is characterized by the degeneration of retinal ganglion cells (RGCs) and their axons (1-4). It is estimated that, by $2020,>80$ million people will be affected worldwide, with at least 6 to 8 million of them becoming bilaterally blind (5). Current glaucoma therapies target the reduction of intraocular pressure (IOP), but since RGC death is the cause of irreversible vision loss, neuroprotection may be an effective strategy for glaucoma treatment (5). Various methods have been used to establish animal models for the study of glaucoma. Due to elevated intraocular pressure (IOP) being well recognized as the sole modifiable risk factor for the development of glaucoma in the majority of cases $(3,6-13)$, the establishment of animal models with chronic elevated IOP is favorable for simulating the pathogenesis of glaucoma.

Elevated IOP is typically caused by excessive aqueous humor production, aqueous humor outflow resistance or increased episcleral vein pressure (14). Different methods of targeting the latter two pathways, in order to cause elevated IOP, have emerged, each with benefits and limitations. For example, cautery of the episcleral vessels may damage the intraocular hemal circumfluence, leading to increased congestive IOP, but it may also cause ocular ischemic disease (15-17). Episcleral vein sclerosis induced by hypertonic PBS (18) is considered to be a suitable model for chronic elevated IOP in rodents $(19,20)$, but imposes technical issues due to the injection procedure and the non-uniformity of the elevation in IOP. Direct burning of the trabecular meshwork with a laser is another effective way of increasing IOP, but it requires advanced technical skills and repeated laser photocoagulation of the trabecular meshwork or the trabecular meshwork and episcleral veins $(21,22)$. Therefore, to the best of our knowledge, no comprehensive animal models of glaucoma currently exist.

A microbead method for injecting substances into the anterior chamber of the eye to create a trabecular meshwork 
channel blockade was developed by exploiting methods used for injecting ghost red blood cells (23) and latex microspheres (24-28). This injection raises the IOP and is accompanied by the degeneration of RGCs and axons $(17,29)$. However, repeated injections increase the risk of hitting and damaging intraocular tissues and causing interexperimental variability. The present study aimed to improve this microbead injection procedure to achieve an animal model of glaucoma that exhibits a longer and more consistent elevation in IOP.

The construction of an animal model of chronic glaucoma to enable the study of the degree and pattern of RGCs loss would ideally incorporate the ability for continuous observation. However, the quantification of RGCs is most commonly performed by histological analysis of fixed retinal tissue, which is laborious and requires the sacrifice of animals for analysis at each time point. This limitation may be overcome in mouse models by the use of confocal scanning laser ophthalmoscopy (cSLO) and the fluorescent protein-labeling of RGCs (30). The aim of the present study was to characterize and compare the RGC loss in Thy1-cyan fluorescent protein (Thy1-CFP) -expressing mice and C57BL/6 mice.

The present study evaluated a modified intracameral microsphere injection technique, in which microspheres are suspended in an ophthalmic viscosurgical device (OVD) to induce IOP elevation in C57BL/6 and Thy1-CFP mice. Whether this modified approach may effectively produce a mouse glaucoma model with chronic and sustained IOP elevation was investigated. In addition, the RGC loss observed in Thyl-CFP mice and C57BL/6 mice was compared.

\section{Materials and methods}

Animals. A total of 91 mice (86 C57BL/6 and 5 Thy1-CFP mice purchased from the Animal House of Central South University, Changsha, China). The Thy1-CFP mice were also C57BL/6 mice) aged between 12 and 20 weeks old and weighing 25-40 g were used in the present study; Both male and female were used for analysis ( $\mathrm{n}=86$ C57BL/6 51 male, 35 female; $n=5$ Thy1-CFP, 5 male). The mice were housed in a standard animal room, with food and water provided ad libitum. A constant environment was maintained, with a 12-h light/dark cycle (illumination time 6:00-18:00), a relative humidity of $50 \pm 5 \%$, a temperature of $20 \pm 3^{\circ} \mathrm{C}$ and $40 \pm 10 \mathrm{db}$ background noise. All experimental procedures adhered to the ARVO (Association of Research in Vision and Ophthalmology) Statement (31) for the Use of Animals in Ophthalmic and Vision Research. The present study was approved by the Ethics Committee of the Second Xiangya Hospital of Central South University (Changsha, China).

Groupings. The mice were allocated into four experimental groups, which were injected with different mixtures into the anterior chamber in right eye. Experimental group A (microbead) contained 34 randomly allocated C57BL/6 mice, which were injected with $2 \mu \mathrm{l}$ of $15 \mu \mathrm{m}$ microbeads (10x $10^{6}$ beads/ml; FluoSpheres Polystyrene Microspheres; cat. no. F21012; Thermo Fisher Scientific, Inc., Waltham, MA, USA) and $1 \mu 1$ of air. Experimental group B [microbeads + hydroxypropyl methylcellulose (HPM)] contained 33 randomly allocated C57BL/6 mice, which were injected with $2 \mu \mathrm{l}$ of the microbeads $\left(10 \times 10^{6}\right.$ beads $\left./ \mathrm{ml}\right)$ and $1 \mu \mathrm{l}$ of $2 \%$ HPM (Methocel; Novartis Pharma S.A.S., Rueil-Malmaison, France). Control group $\mathrm{C}$ (control) contained the remaining 19 C57BL/6 mice, which received an injection of $2 \mu 1$ PBS and $1 \mu \mathrm{l}$ air. The live imaging group contained the 5 Thy1-CFP mice, which received injections of $2 \mu 1$ microbeads (10x10 6 beads/ml) and $1 \mu \mathrm{l} \mathrm{HPM.}$

Microbead injection. The mice were injected as described previously (32) with modifications. Prior to surgery, the mice were anesthetized with an intraperitoneal injection of Avertin (0.5 ml/20 g; Sigma-Aldrich; Merck KGaA, Darmstadt, Germany) and the local application of $0.5 \%$ proparacaine hydrochloride ophthalmic solution (Bausch \& Lomb Pharmaceuticals, Tampa, FL, USA) to each eye. Subsequently, $0.5 \%$ tropicamide ophthalmic solution (Bausch \& Lomb Pharmaceuticals) was used to dilate the pupils of both eyes.

Anterior chamber paracentesis was performed using a sterile 32-gauge needle. A corneal incision was made and the aqueous humor left to drain out as much as possible in order to obtain enough space for microbeads. A Hamilton syringe (Hamilton Company, Reno, NV, USA) was used to inject the microbeads + air, microbead + HPM or PBS + air. The fluid was injected slowly and steadily into the anterior chamber over $60 \mathrm{sec}$, and the empty syringe was left in the anterior chamber for a further $60 \mathrm{sec}$. Following surgery, $0.5 \%$ erythromycin ophthalmic ointment (Bausch \& Lomb Pharmaceuticals) was applied to each eye.

Animal acclimatization and IOP measurements. The method used for animal acclimatization and IOP measurements has been described previously (32). Animal behavioral training was completed in two phases. In the 1-week-long initial phase, the mice were held in the experimenter's hands for 15-20 min every day and acclimated to the dropping of anesthetic or PBS into each eye. In the 10-day-long second phase, the mice were acclimatized to daily IOP measurements under topical anesthesia by the application of $0.5 \%$ proparacaine hydrochloride ophthalmic solution (Bausch \& Lomb Pharmaceuticals, Tampa, FL, USA). A TonoLab rebound tonometer (Icare TONOLAB tonometer; Colonial Medical Supply, Windham, $\mathrm{NH}$, USA) and topical anesthesia were used to obtain noninvasive IOP measurements, which were performed at the same time every day to avoid pressure fluctuations associated with circadian rhythm and light/dark cycles (33). The daily experimental IOP measurements began $24 \mathrm{~h}$ after the first microbead injection and continued for 1 month.

Anterior segment imaging. Microbead distribution was recorded 3 days after injection by obtaining anterior segment images with a High Definition CMOS Color camera (Olympus Corporation, Tokyo, Japan) attached to a DMRA2 fluorescent microscope (Leica Micro Systems, Ltd., Milton Keynes, UK; magnification, $\mathrm{x} 200)$. The mice were kept under general anesthesia (Avertin; $0.5 \mathrm{ml} / 20 \mathrm{~g}$ ) during imaging.

Frozen sectioning for anterior chamber angle measurements, retinal histology and immunohistochemistry. From each group, mice were randomly selected and sacrificed by an overdose of $\mathrm{CO}_{2}$ or 6 weeks after the initial injections. Mice were 
B

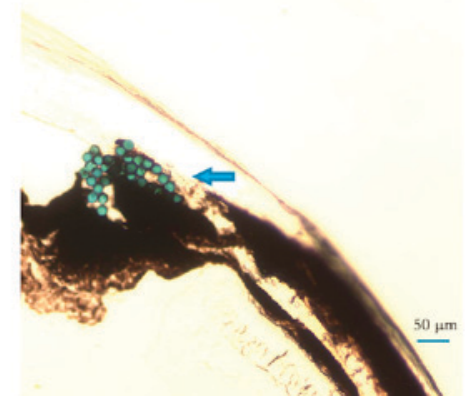

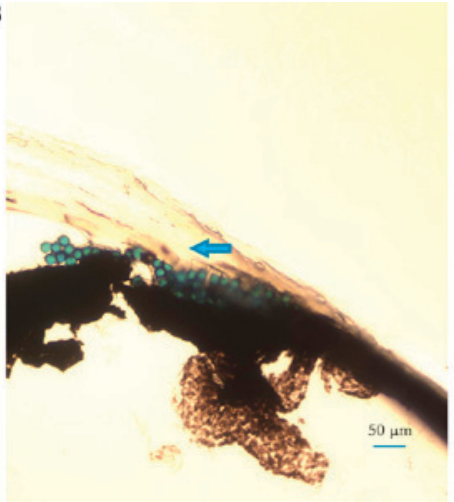

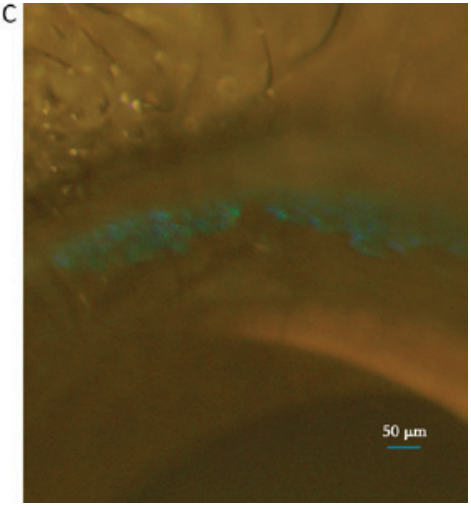

Figure 1. Distribution of microbeads in the anterior chamber of the eye following injection. (A) Microbead injection group. (B) Microbeads + HPM injection group. More microbeads entered the Schlemm's canal in the microbeads + HPM injection group. (C) Accumulation of microbeads in the anterior chamber angle and Schlemm's canal (arrows) in the microbeads + HPM group. Magnification, x200; scale bar=50 $\mu \mathrm{m}$. HPM, hydroxypropyl methylcellulose.

placed into a transparent $50 \times 26 \times 20 \mathrm{~cm}$ chamber and $100 \%$ $\mathrm{CO}_{2}$ were infused into the chamber at $6 \mathrm{lpm}$ rate. Once mice had ceased to breathe, they were left for at least 1 min before being removed from the chamber and the absence of heartbeat confirmed mice had succumbed to $\mathrm{CO}_{2}$ overdose. The eyes were removed quickly by pinching off the optic nerve and then washed in PBS three times (10 min/wash). The eyeballs were dissected into two parts $1 \mathrm{~mm}$ behind the corneal limbus. The anterior segments were coated with optimum cutting temperature reagent (Sakura Finetek USA, Inc., Torrance, CA, USA) and then snap-frozen in liquid nitrogen and cut into $50-\mu \mathrm{m}$-thick sections. Images of the anterior chamber angle sections were obtained with a bright-field microscope High Definition CMOS Color camera.

The retinas were separated from the posterior segments and placed in $4 \%$ paraformaldehyde fixative (Electron Microscopy Sciences, Hatfield, PA, USA) for $30 \mathrm{~min}$, rinsed three times with PBS (10 min/wash) and then incubated in 30\% sucrose solution overnight at $4{ }^{\circ} \mathrm{C}$. The samples were again rinsed three times in PBS (10 min/wash) and blocked with $10 \%$ normal donkey serum diluted in PBS (Jackson Inmuno Research Labs, West Grove, PA, USA) for $8 \mathrm{~h}$ at $4^{\circ} \mathrm{C}$. Goat anti-POU domain class 4 transcription factor 2 (Brn3b: A RGC specific marker (34); catalog no. sc-31989; Santa Cruz Biotechnology, Inc., Dallas, TX, USA) primary antibody was diluted 1:100 in PBS and donkey anti-goat Texas Red secondary antibody (Molecular Probes; Thermo Fisher Scientific, Inc.) was diluted 1:50 in 10\% normal donkey serum (Jackson Inmuno Research Labs). The retinas were incubated for 6 days at $4^{\circ} \mathrm{C}$ with the primary antibody, washed three times in PBS (10 $\mathrm{min} /$ wash). Subsequently, the retinas were incubated for $24 \mathrm{~h}$ at $4^{\circ} \mathrm{C}$ with the secondary antibody, washed three times in PBS (10 min/wash) and flat-mounted on a glass slide with a coverslip (Thermo Scientific Shandon ColorFrost Plus slides; Thermo Fisher Scientific, Inc.).

Each retina was divided into four optic-nerve-centered sections. From each quadrant, three areas were selected, at $1 / 6,1 / 2$ and $5 / 6$ retinal radiuses away from the optic nerve. Images were obtained with a 10x20 fluorescence microscope (DMI6000B; Leica Micro Systems, Ltd.). All Brn3b-positive RGCs in each area were counted. The percentage of RGC loss was calculated as follows: RGC loss $(\%)=($ number of RGCs in experimental group eyes/number of RPGs in control group eyes) $\mathrm{x} 100$. The number of Brn3b-positive RGCs was counted manually using the Cell Counter plugin for Image $\mathbf{J}$ (Image J $1.43 \mathrm{u}$; National Institutes of Health, Bethesda, MD, USA).

Live imaging by confocal scanning laser microscopy. The Thy1-CFP mice were anesthetized with isoflurane (2\%; MWI Veterinary Supply, Inc., Meridian, ID, USA) in $95 \% \mathrm{O}_{2}$ and $5 \% \mathrm{CO}_{2}$, and placed on a temperature-controlled heated platform at $38^{\circ} \mathrm{C}$. Topical tropicamide and $1 \%$ atropine sulfate ophthalmic solution (both Bausch \& Lomb Pharmaceuticals) were used to dilate the pupils. A drop of hypromellose (2.5\%; Akorn Pharmaceuticals, Lake Forest, IL, USA) was placed in the eye and a glass coverslip was placed over it to create a planoconcave lens on the mouse cornea for in vivo imaging. An LSM 5 Exciter Laser scanning confocal system (Carl Zeiss Inc., Thornwood, NY, USA) was modified (using an Axioskop 2 upright microscope equipped with a LSM 5 Exciter Laser scanning confocal system) to visualize CFP [blue-light cSLO (bCSLO); $460 \mathrm{~nm}$ excitation and $490 \mathrm{~nm}$ detection]. The number of $\mathrm{CFP}^{+} \mathrm{RGCs}$ in the same area of the retina were assessed in vivo once a week for 6 weeks.

Statistical analysis. Data were recorded in Microsoft Excel 2003 (Microsoft Corporation, Redmond, WA, USA) and analyzed using the Student's t-test $(\mathrm{a}=0.05)$. Data are expressed as the mean \pm standard deviation. $\mathrm{P}<0.05$ was considered to indicate a statistically significant difference.

\section{Results}

Microbeads block the outflow of aqueous humor. The present study adopted and modified a method of inducing elevated IOP by injecting the anterior chamber of the eye with microbeads and HPM. The injected microbeads accumulated at the angle of the anterior chamber, blocking the outflow of aqueous humor (Fig. 1A and B). Vertical sections through the anterior segment revealed microbeads clustered near the iridocorneal angle (Fig. 1A and B). These observations demonstrate that the microbeads moved with the aqueous humor towards the trabecular meshwork. In addition, more microbeads entered the Schlemm's canal in the microbead + HPM injection group 


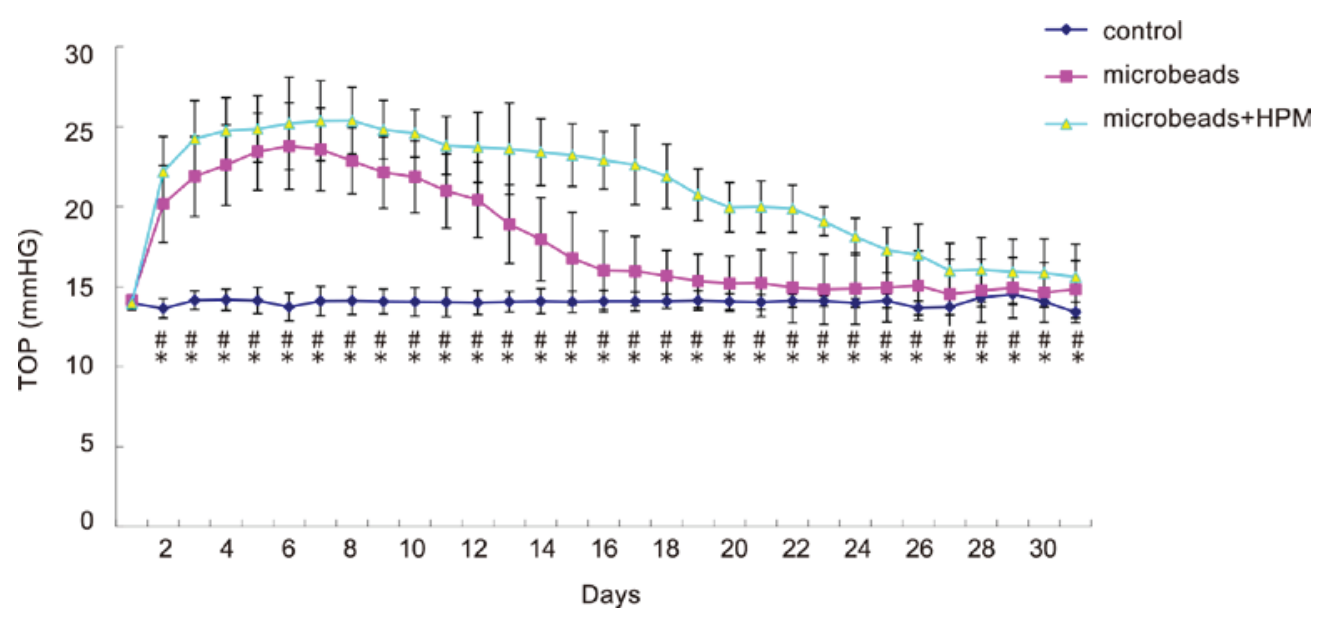

Figure 2. Assessment of IOP elevation following the injection of microbeads or microbeads + HPM. Microbeads and microbeads + HPM injection induced IOP elevation, but the microbeads + HPM injections induced a longer and higher peak of IOP elevation compared with microbeads alone. Compared with the control group, an elevation in IOP was induced within 2 days after injection in the microbeads and microbeads + HPM groups. Data are presented as mean \pm standard deviation. ${ }^{*} \mathrm{P}<0.05$ vs. microbeads group. ${ }^{*} \mathrm{P}<0.05$ vs. microbeads + HPM group. Magnification, $\mathrm{x} 200$. IOP, intraocular pressure; HPM, hydroxypropyl methylcellulose.

compared with the group injected with microbeads alone (Fig. 1A and B). No inflammatory responses (opaque cornea, edema, iris exudation or cloudy anterior chamber) or overt damage were observed in the anterior chamber of the eye in mice injected with microbeads alone or microbeads + HPM (Fig. 1).

Microbead injection significantly increases the IOP of mice. The IOP of the mice as measured every day following the microbead injections. The control mice that received an injection of PBS exhibited a steady IOP level $(14.1 \pm 0.2 \mathrm{~mm} \mathrm{Hg})$ throughout the experimental period. Compared with the control group, an elevation in IOP was induced within 2 days after injection in the microbead and microbeads + HPM groups. There were significant differences in the microbead group compared with the control on days 2-31 $(\mathrm{P}<0.05$; Fig. 2), and there were also significant differences between the microbeads + HPM group and control on days 2-31 ( $\mathrm{P}<0.05$; Fig. 2). The elevated IOP levels were maintained for $\sim 2$ weeks, reaching a peak of $23.8 \pm 2.7 \mathrm{mmHg} 5-7$ days after injection (Fig. 2). The mean IOP in the microbead group was $18.0 \pm 3.4 \mathrm{mmHg}$ throughout the experimental period from day $0-31$, which was significantly increased by 1.3 times, compared with the control group $(18.0 \pm 3.4 \mathrm{mmHg}$ vs. $14.0 \pm 0.2 \mathrm{mmHg} ; \mathrm{P}<0.05)$.

Microbead + HPM injection is significantly more effective compared with microbeads alone for elevating IOP. An injection of microbeads + HPM induced an IOP elevation that lasted for 4 weeks, 2 weeks longer compared with that observed in the microbead alone group. For the first 7 days after injection, the microbeads + HPM group exhibited IOP elevation kinetics that were similar compared with those of the microbead-injected group. However, following day 7, the IOP level in the microbead + HPM injected group continued to increase, reaching a peak of $25.4 \pm 2.1 \mathrm{mmHg} 7-8$ days after injection (Fig. 2). The mean IOP in the microbeads + HPM group was $21.3 \pm 3.4 \mathrm{mmHg}$ during the experimental period, which was significantly increased by 1.5 times compared with the control group $(21.3 \pm 3.4 \mathrm{mmHg}$ vs. $14.0 \pm 0.2 \mathrm{mmHg} ; \mathrm{P}<0.05)$. All 67 mice that received microbead or microbead + HPM injections into the anterior chamber of the eye exhibited significant IOP elevation when compared with the PBS-injected control group $(\mathrm{P}<0.05)$. This indicates that injection of microbeads or microbeads + HPM into the anterior chamber of the eye effectively induces a durative and reversible elevation of IOP in mice. The microbead + HPM injection was more effective compared with microbeads alone, as it induced a longer and higher peak of IOP elevation compared with the injection with microbeads alone.

$R G C$ death is increased by microbead and microbead + HPM injection. RGC loss, another hallmark of glaucoma, was evaluated by quantifying RGC numbers in a retinal whole mount following the elevation of IOP. The mice injected with microbeads or microbeads + HPM were sacrificed 3 and 6 weeks following the initial injection. The Brn3b labeling revealed a reduction in the number and density of RGCs in the eyes that were injected with microbeads or microbeads + HPM when compared with the control group (Fig. 3A). IOP elevation induced a significant reduction in RGC density (Fig. 3B, C, E and F). The analysis of RGC density in microbead or microbeads + HPM groups demonstrated that IOP elevation induced RGC death and that the extent of RGC death was greater in eyes injected with microbeads + HPM compared with microbeads alone when the whole retina was taken into account. The extent of RGC death following the injection of microbeads was $14.2 \pm 0.8 \%$ at 3 weeks and $25.0 \pm 1.3 \%$ at 6 weeks (Fig. 3G). RCG death following injection of microbeads + HPM was $19.1 \pm 1.3 \%$ at 3 weeks and $33.2 \pm 1.9 \%$ at 6 weeks (Fig. 3G). Thus, RGC death was significantly higher in the microbeads + HPM at 3 weeks and 6 weeks after injection compared with the microbead group (both $\mathrm{P}<0.05$ ).

Microbead + HPM injection technique allows the in vivo assessment of RGC numbers in Thyl-CFP mice. The minimally invasive nature of the technique used in the present 

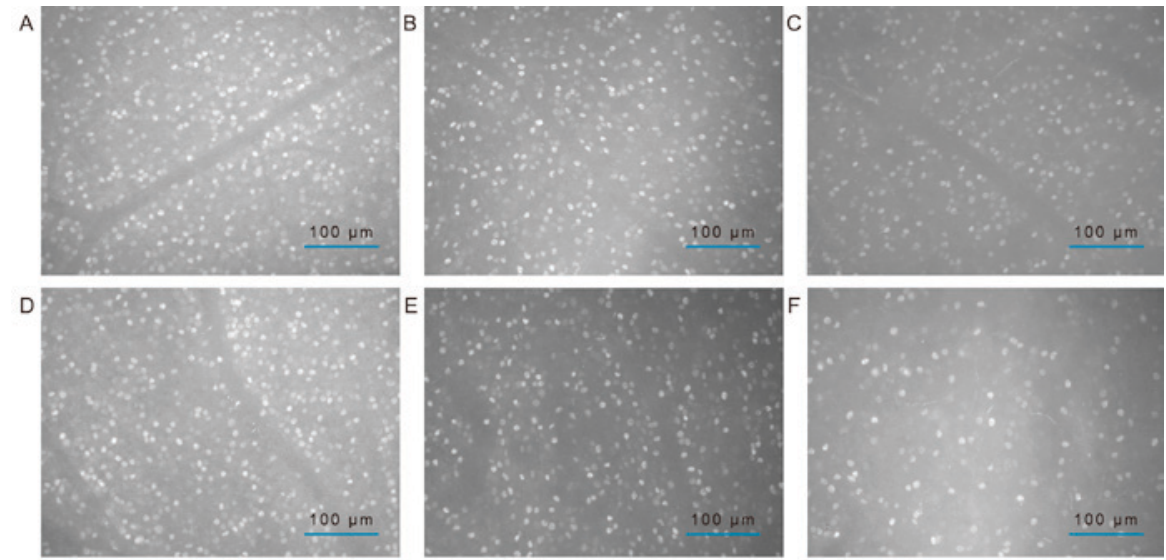

G

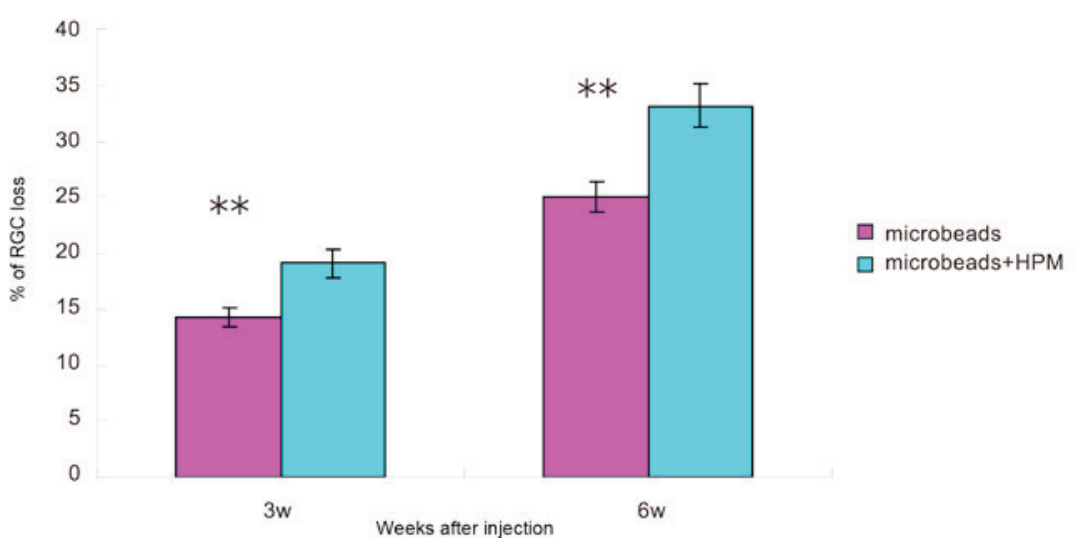

Figure 3. Quantification of RGC loss in mice following the injection of microbeads or microbeads + HPM. Distribution of Brn3b ${ }^{+}$RGCs in the ganglion cell layer of the retina in the (A) control group 6 weeks after injection, (B) microbeads group 3 weeks after injection, (C) microbeads group 6 weeks after injection, (D) control group 6 weeks after injection, (E) microbeads + HPM group 3 weeks after injection. and (F) microbeads + HPM group 6 weeks after injection. (G) Quantification of RGC loss 3 and 6 weeks after injection. Scale bar $=100 \mu \mathrm{m}$. RGC death was significantly higher in the microbeads + HPM at 3 weeks and 6 weeks after injection compared with the microbeads group. ${ }^{* *} \mathrm{P}<0.05$ vs. microbeads + HPM group. Magnification, $\mathrm{x} 200$. RGC, retinal ganglion cells; HPM, hydroxypropyl methylcellulose.
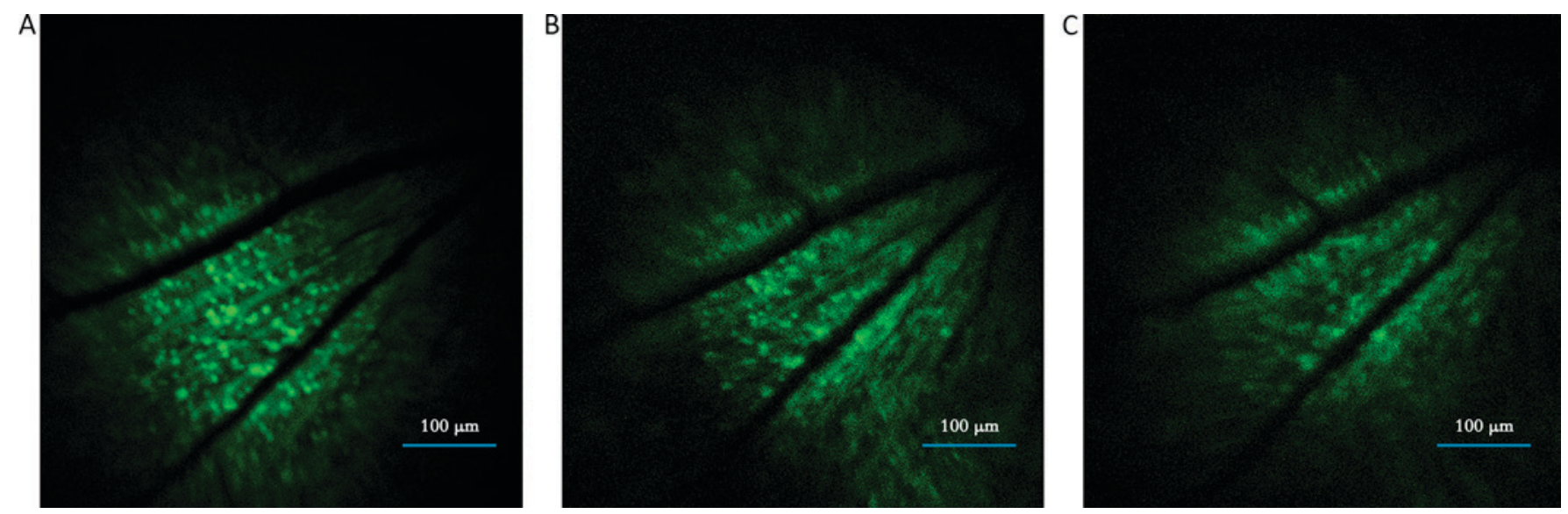

Figure 4. In vivo imaging of RGC loss in Thyl-CFP mice following the injection of microbeads + HPM. In vivo imaging of CFP (A) prior to microbeads + HPM injection, (B) 3 weeks after injection and (C) 6 weeks after injection. Scale bar=100 $\mu \mathrm{m}$. The number of CFP+RGCs in the same area of the retina was assessed in vivo and a progressive decrease in the number of fluorescent points was detected using serial blue-light confocal scanning laser ophthalmoscopy images following IOP elevation. Magnification, x200. RGC, retinal ganglion cells; CFP, cyan fluorescent protein; HPM, hydroxypropyl methylcellulose; IOP, intraocular pressure.

study permitted the assessment of the same mice at different time points. Progressive decreases in the number of $\mathrm{CFP}^{+}$ RCGs in the serial bCSLO images following IOP elevation (Fig. 4). $\mathrm{CFP}^{+} \mathrm{RGC}$ loss was $24.3 \pm 2.3 \% 3$ weeks after the elevation of IOP by a single injection and the number of
CFP-positive RGCs continued to decrease over time; 6 weeks after, $\mathrm{CFP}^{+} \mathrm{RGC}$ loss was $39.3 \pm 1.7 \%$ (Fig. 5). These results were comparable with the results obtained from immunohistochemical staining of the C57BL/6 mice with elevated IOP (Fig. 5). 


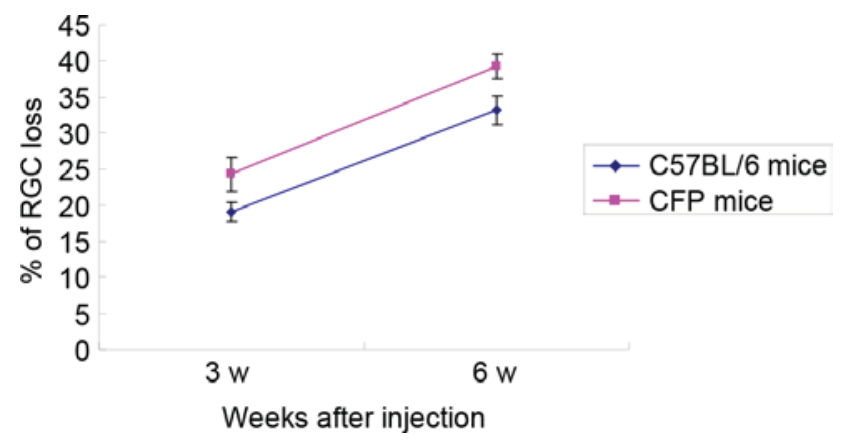

Figure 5. Percentage of RGC loss in Thy1-CFP mice measured by in vivo imaging is similar compared with that observed in C57BL/6 mice with in vitro immunohistochemistry following the injection of microbeads + hydroxypropyl methylcellulose. RGC, retinal ganglion cells; CFP, cyan fluorescent protein.

\section{Discussion}

Injecting microbeads or microbeads + HPM into the anterior chamber of the eye obstructs aqueous humor outflow through the trabecular meshwork and leads to a gradual increase in IOP (35-37). In the present study, more microbeads entered the Schlemm's canal in the microbead + HPM injection group compared with the microbead alone group. This suggests that HPM serves to immobilize the microbeads at the anterior chamber angle. While blocking the trabecular network, the converged microbeads may also cause trabecular meshwork swelling and an inflammatory reaction, which may elevate IOP or lead to an anterior chamber reaction, although this was not observed in the present study.

The microbead-induced ocular hypertension mouse model imitates the clinical conditions of pseudoexfoliation-associated glaucoma and ghost cell glaucoma $(38,39)$, in addition to leaving a cleared visual axis that allows easy observation of the retina and optic disc.

The diameter of the microbeads used in the present experiment was $15 \mu \mathrm{m}$, which is smaller than the initial interlaminar spaces of the trabecular meshwork $(25-75 \mu \mathrm{m})$, but larger than the inner pores of the trabecular meshwork (0.2-2 $\mu \mathrm{m})(40)$. A preliminary test conducted by Samsel et al (35), using 1-2 $\mu \mathrm{m}$ beads, failed to demonstrate a consistent increase in IOP and guiding the beads into the anterior chamber proved difficult; changing to a bead size of $5 \mu \mathrm{m}$ resulted in sustainable ocular hypertension. Chen et al (41) revealed that injecting the anterior chamber with $10 \mu \mathrm{m}$ beads induced a longer duration and higher peak value of IOP elevation when compared with $15 \mu \mathrm{m}$ beads. A potential explanation for this may be that the $10 \mu \mathrm{m}$ beads were able to enter the Schlemm's canal more easily compared with $15 \mu \mathrm{m}$ beads. However, the advantages of the long-term induction of ocular hypertension by the larger beads remains to be elucidated (17).

The present study selected $15 \mu \mathrm{m}$ microbeads based on preliminary studies by our group that evaluated 10 and $15 \mu \mathrm{m}$ microbeads (data not shown). During the surgical procedure, the volume of each injection was limited to $3 \mu \mathrm{l}$ and the microbeads were injected into the anterior chamber slowly (over $60 \mathrm{sec}$ ) in order to minimize acute IOP elevation. Although fluctuations in IOP are inevitable, previous studies indicate that injection volume determines the magnitude of IOP elevation in rats (29), and that different levels and durations of elevated IOP may be obtained by altering the frequency and number of microbeads injected (24). The present study aimed to investigate the effectiveness of HPM injections in an animal glaucoma model. The injection was conducted only once in order to reduce the likelihood of errors and unnecessary injury that repeated injections may cause.

The results of the present study demonstrated that injecting microbeads alone and microbeads + HPM increased IOP compared with the control group. However, compared with microbeads alone, IOP was higher and more stable in the mice that received microbeads + HPM. HPM is a biologically inert substance with a low molecular weight, low surface tension and high viscosity. It is a type of dispersive OVD that is widely used in cataract surgery to maintain the intraocular space. Incomplete removal of an OVD may lead to aqueous humor outflow blockage and IOP elevation. Kocak-Altintas et al (42) reported that OVDs with higher viscosity were associated with a higher incidence of postoperative IOP, potentially because OVDs with a high viscosity tend to be harder to remove. Oshika et al (43) revealed that OVDs of different viscosities remained in the porcine anterior chamber for different periods of time.

In the current study, the microbeads + HPM group had higher IOP levels and a greater RGC reduction compared with the microbead alone group. This suggests that HPM blocks the aqueous humor outflow due to its biologically inert and viscous characteristics. The retention of HPM in the anterior chamber helped immobilize the microbeads at the anterior chamber angle. The microbeads were the primary contributors to blockage of the anterior chamber angle and caused sustained IOP elevation.

The present study identified a significant reduction in RGC cell density in the retinas of the microbeads + HPM and microbead alone groups, as confirmed by in vivo imaging in Thy1-CFP mice. This result is in agreement with the results obtained from pig models $(16,44)$. The lack of any observed RGC loss following PBS injection suggests that the sustained IOP elevation, rather than any transitory IOP increase, contributed to the loss of RGCs. Furthermore, the results of in vivo imaging revealed a similar decline in RGCs compared with the C57BL/6 mice samples at the same time points, suggesting that it provides an accurate measure of RGCs. In vivo imaging is a more convenient and repeatable procedure for use in experimental animals, specifically in studies of neuroprotection. Statistical evidence indicates that $30 \%$ of Thyl-expressing cells lack recordable CFP fluorescence; however, this does not limit the accuracy of the overall results (45).

In conclusion, the microbead and HPM injection technique used in the present study successfully produced a chronic elevation in IOP in mice. This technique is simple and cost-effective, in addition to allowing the in vivo observation of RGC numbers. The mice injected with a combination of microbeads and HPM exhibited higher IOP elevations and greater reductions in RGCs compared with mice injected with microbeads alone. The in vivo imaging revealed a similar degree of RGC loss when compared with the in vitro results, suggesting that the in vivo imaging provides an accurate measure of RGCs. In vivo imaging is more convenient and permits long-term experiments, which are particularly important for the study of neuroprotection. It is a valuable tool for 
monitoring RGC changes over time in various mice model of RGC loss. This approach will be useful for studying the pathology of optic neuropathy including glaucoma and for developing new therapies.

\section{Acknowledgements}

The present study was supported by the National Natural Science Foundation of China (Beijing, China; grant no. $81,300,758)$.

\section{References}

1. Carelli V, Ross-Cisneros FN and Sadun AA: Optic nerve degeneration and mitochondrial dysfunction: Genetic and acquired optic neuropathies. Neurochem Int 40: 573-584, 2002.

2. Lorenzetto E, Ettorre M, Pontelli V, Bolomini-Vittori M, Bolognin S, Zorzan S, Laudanna C and Buffelli M: Rac1 selective activation improves retina ganglion cell survival and regeneration. PLoS One 8: e64350, 2013.

3. Feng L, Zhao Y, Yoshida M, Chen H, Yang JF, Kim TS, Cang J, Troy JB and Liu X: Sustained ocular hypertension induces dendritic degeneration of mouse retinal ganglion cells that depends on cell type and location. Invest Ophthalmol Vis Sci 54: 1106-1117, 2013.

4. Vidal-Sanz M, Salinas-Navarro M, Nadal-Nicolás FM, Alarcón-Martínez L, Valiente-Soriano FJ, de Imperial JM, Avilés-Trigueros M, Agudo-Barriuso M and Villegas-Pérez MP: Understanding glaucomatous damage: Anatomical and functional data from ocular hypertensive rodent retinas. Prog Retin Eye Res 31: 1-27, 2012.

5. Quigley HA and Broman AT: The number of people with glaucoma worldwide in 2010 and 2020. Br J Ophthalmol 90: 262-267, 2006.

6. Feng L, Chen H, Suyeoka G and Liu X: A laser-induced mouse model of chronic ocular hypertension to characterize visual defects. J Vis Exp: doi: 10.3791/50440.

7. Zhong L: A modified chronic ocular hypertension rat model for retinal ganglion cell neuroprotection. Front Med 7: 367-377, 2013

8. Buie LK, Karim MZ, Smith MH and Borrás T: Development of a model of elevated intraocular pressure in rats by gene transfer of bone morphogenetic protein 2. Invest Ophthalmol Vis Sci 54 $5441-5455,2013$

9. Lichter PR: Impact of intraocular pressure reduction on glaucoma progression. JAMA 288: 2607-2608, 2002.

10. Heijl A, Leske MC, Hyman L, Yang Z and Bengtsson B; EMGT Group: Intraocular pressure reduction with a fixed treatment protocol in the Early Manifest Glaucoma Trial. Acta Ophthalmol 89: 749-754, 2011.

11. Khan AK, Tse DY, van der Heijden ME, Shah P, Nusbaum DM, Yang Z, Wu SM and Frankfort BJ: Prolonged elevation of intraocular pressure results in retinal ganglion cell loss and abnormal retinal function in mice. Exp Eye Res 130: 29-37, 2015.

12. Matsumoto Y, Kanamori A, Nakamura M and Negi A: Rat chronic glaucoma model induced by intracameral injection of microbeads suspended in sodium sulfate-sodium hyaluronate. Jpn J Ophthalmol 58: 290-297, 2014

13. Kass MA, Heuer DK, Higginbotham EJ, Johnson CA, Keltner JL, Miller JP, Parrish RK II, Wilson MR and Gordon MO: The ocular hypertension treatment study: A randomized trial determines that topical ocular hypotensive medication delays or prevents the onset of primary open-angle glaucoma. Arch Ophthalmol 120 701-713, 829-830, 2002.

14. Tektas OY and Lütjen-Drecoll E: Structural changes of the trabecular meshwork in different kinds of glaucoma. Exp Eye Res 88: 769-775, 2009.

15. Shareef SR, Garcia-Valenzuela E, Salierno A, Walsh J and Sharma SC: Chronic ocular hypertension following episcleral venous occlusion in rats. Exp Eye Res 61: 379-382, 1995.

16. Ruiz-Ederra J, García M, Hernández $M$, Urcola $H$, Hernández-Barbáchano E, Araiz J and Vecino E: The pig eye as a novel model of glaucoma. Exp Eye Res 81: 561-569, 2005.

17. Urcola JH, Hernández M and Vecino E: Three experimental glaucoma models in rats: Comparison of the effects of intraocular pressure elevation on retinal ganglion cell size and death. Exp Eye Res 83: 429-437, 2006.
18. Morrison JC, Moore CG, Deppmeier LM, Gold BG, Meshul CK and Johnson EC: A rat model of chronic pressure-induced optic nerve damage. Exp Eye Res 64: 85-96, 1997.

19. Nissirios N, Chanis R, Johnson E, Morrison J, Cepurna WO, Jia L, Mittag T and Danias J: Comparison of anterior segment structures in two rat glaucoma models: An ultrasound biomicroscopic study. Invest Ophthalmol Vis Sci 49: 2478-2482, 2008.

20. Morrison JC: Elevated intraocular pressure and optic nerve injury models in the rat. J Glaucoma 14: 315-317, 2005.

21. Biermann J, van Oterendorp C, Stoykow C, Volz C, Jehle T, Boehringer D and Lagrèze WA: Evaluation of intraocular pressure elevation in a modified laser-induced glaucoma rat model. Exp Eye Res 104: 7-14, 2012

22. Levkovitch-Verbin H, Quigley HA, Martin KR, Valenta D, Baumrind LA and Pease ME: Translimbal laser photocoagulation to the trabecular meshwork as a model of glaucoma in rats. Invest Ophthalmol Vis Sci 43: 402-410, 2002.

23. Quigley HA and Addicks EM: Chronic experimental glaucoma in primates. I. Production of elevated intraocular pressure by anterior chamber injection of autologous ghost red blood cells. Invest Ophthalmol Vis Sci 19: 126-136, 1980.

24. Weber AJ and Zelenak D: Experimental glaucoma in the primate induced by latex microspheres. J Neurosci Methods 111: 39-48, 2001.

25. Peiffer RL, Popovich KS and Nichols DA: Long-term comparative study of the Schocket and Joseph glaucoma tube shunts in monkeys. Ophthalmic Surg 21: 55-59, 1990.

26. Ernest JT and Potts AM: Pathophysiology of the distal portion of the optic nerve. II. Vascular relationships. Am J Ophthalmol 66: 380-387, 1968.

27. Paula JS, Ribeiro VR, Sampaio RB, Mendonca RJ, Haddad A, Tedesco AC, Coutinho-Netto J, Haendchen HA and Jorge R: Rabbit rubeosis iridis induced by intravitreal latex-derived angiogenic fraction. Curr Eye Res 36: 857-859, 2011.

28. Matsumoto Y and Johnson DH: Trabecular meshwork phagocytosis in glaucomatous eyes. Ophthalmologica 211: 147-152, 1997.

29. Sappington RM, Carlson BJ, Crish SD and Calkins DJ: The microbead occlusion model: A paradigm for induced ocular hypertension in rats and mice. Invest Ophthalmol Vis Sci 51: 207-216, 2010.

30. Leung CK, Lindsey JD, Crowston JG, Ju WK, Liu Q, Bartsch DU and Weinreb RN: In vivo imaging of murine retinal ganglion cells. J Neurosci Methods 168: 475-478, 2008.

31. 'Statement for the Use of Animals in Ophthalmic and Visual Research', The Association for Research in Vision and Ophthalmology, USA. http://www.arvo.org/About_ARVO/Policies/Statement_for_ the Use of Animals in_Ophthalmic and Visual_Research/. Accessed October, 2013.

32. Ding C, Wang P and Tian N: Effect of general anesthetics on IOP in elevated IOP mouse model. Exp Eye Res 92: 512-520, 2011.

33. Jia L, Cepurna WO, Johnson EC and Morrison JC: Effect of general anesthetics on IOP in rats with experimental aqueous outflow obstruction. Invest Ophthalmol Vis Sci 41: 3415-3419, 2000.

34. Badea TC and Nathans J: Morphologies of mouse retinal ganglion cells expressing transcription factors Brn3a, Brn3b, and Brn3c: Analysis of wild type and mutant cells using genetically-directed sparse labeling. Vision Res 51: 269-279, 2011.

35. Samsel PA, Kisiswa L, Erichsen JT, Cross SD and Morgan JE: A novel method for the induction of experimental glaucoma using magnetic microspheres. Invest Ophthalmol Vis Sci 52: 1671-1675, 2011.

36. Swaminathan SS, Oh DJ, Kang MH, Ren R, Jin R, Gong H and Rhee DJ: Secreted protein acidic and rich in cysteine (SPARC)-null mice exhibit more uniform outflow. Invest Ophthalmol Vis Sci 54: 2035-2047, 2013.

37. Lu Z, Zhang Y, Freddo TF and Gong H: Similar hydrodynamic and morphological changes in the aqueous humor outflow pathway after washout and Y27632 treatment in monkey eyes. Exp Eye Res 93: 397-404, 2011.

38. Konstas AG, Hollo G, Astakhov YS, Teus MA, Akopov EL, Jenkins JN and Stewart WC: Presentation and long-term follow-up of exfoliation glaucoma in Greece, Spain, Russia, and Hungary. Eur J Ophthalmol 16: 60-66, 2006.

39. Spraul CW and Grossniklaus HE: Vitreous hemorrhage. Surv Ophthalmol 42: 3-39, 1997.

40. Inomata H, Bill A and Smelser GK: Aqueous humor pathways through the trabecular meshwork and into Schlemm's canal in the cynomolgus monkey (Macaca irus). An electron microscopic study. Am J Ophthalmol 73: 760-789, 1972. 
41. Chen H, Wei X, Cho KS, Chen G, Sappington R, Calkins DJ and Chen DF: Optic neuropathy due to microbead-induced elevated intraocular pressure in the mouse. Invest Ophthalmol Vis Sci 52: 36-44, 2011.

42. Kocak-Altintas AG, Anayol MA, Cakmak HB and Simsek S: Effects of topical dorzolamide on IOP after phacoemulsification with different types of ophthalmic viscosurgical devices. Eur J Ophthalmol 17: 38-44, 2007.

43. Oshika T, Okamoto F, Kaji Y, Hiraoka T, Kiuchi T, Sato M and Kawana K: Retention and removal of a new viscous dispersive ophthalmic viscosurgical device during cataract surgery in animal eyes. Br J Ophthalmol 90: 485-487, 2006.
44. Garca M, Ruiz-Ederra J,Hernandez-Barbachano H and Vecino E: Topography of pig retinal ganglion cells. J Comp Neurol 486: 361-372, 2005

45. Tosi J, Wang NK, Zhao J, Chou CL, Kasanuki JM, Tsang SH and Nagasaki T: Rapid and noninvasive imaging of retinal ganglion cells in live mouse models of glaucoma. Mol Imaging Biol 12: 386-393, 2010. 